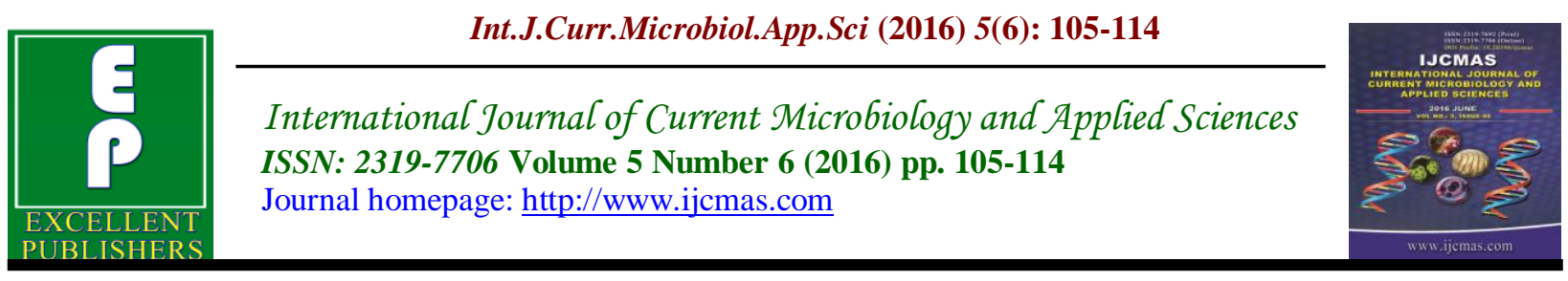

Original Research Article

http://dx.doi.org/10.20546/ijcmas.2016.506.013

\title{
Evaluation of a 12 Disc Test for Phenotypic Detection of $\beta$ - lactamases Resistance in Gram Negative Bacilli
}

\author{
Parul Sinha, Pallawi Goyal, Rajni Sharma*, Aruna Vyas and R.K. Maheshwari \\ Department of Microbiology and Immunology, Sawai Man Singh Medical \\ College, Jaipur, India \\ *Corresponding author
}

\begin{abstract}
A B S T R A C T
Keywords

12 disc test, beta lactamases, AmpC $\beta \mathrm{L}$, co producers.

\section{Article Info}

Accepted:

10 May 2016

Available Online:

10 June 2016

Various mutated forms of $\beta$-lactamases pose a therapeutic challenge to the health care settings as they hydrolyze a wide range of $\beta$-lactam antibiotics. Detection of these enzymes and their co existence is still a diagnostic challenge. In this, we evaluated a novel 12 disc test as a screening test for the presence of any kind of beta lactamases or its co producers. A 12 disc test was put on 300 isolates that showed susceptibility pattern as Intermediate/Resistant to ceftazidime/ ceftriaxone or aztreonam on routine sensitivity testing by modified Kirby bauer method. Further, double disc synergy test, AmpC disc test, E test for MBL and modified Hodge test for KPC/ MBL (used as confirmatory tests) were also done on each of them. By 12 disc test, out of 300 isolates, 64 (21.3\%) were ES $\beta \mathrm{L}, 8(2.6 \%) \mathrm{K} 1 \beta \mathrm{L}$, 76 (25.3\%) high level AmpC $\beta \mathrm{L}, 08(2.6 \%)$ AmpC $\beta \mathrm{L}, 08$ (2.6\%) AmpC $\beta \mathrm{L}+\mathrm{ES} \beta \mathrm{L}$ co producers, 108 (36\%) Carbepenamases and 28 (9.3\%) M $\beta \mathrm{L}+\mathrm{ES} \beta \mathrm{L}$ producers. By confirmatory tests, 64 (21.3\%) were ES $\beta \mathrm{L}, 08$ (2.6\%) AmpC $\beta \mathrm{L}, 64$ (21.3\%) High level AmpC $\beta \mathrm{L}, 08$ (2.6\%) AmpC $\beta \mathrm{L}+\mathrm{ES} \beta \mathrm{L}, 44$ (14.6\%) AmpC $\beta \mathrm{L}+\mathrm{M} \beta \mathrm{L}$, $16(5.3 \%)$ AmpC $\beta \mathrm{L}+\mathrm{M} \beta \mathrm{L}+\mathrm{ES} \beta \mathrm{L}, 52(17.3 \%) \mathrm{M} \beta \mathrm{L}, 8$ (2.6\%) KPC and 12 (4\%) $\mathrm{ES} \beta \mathrm{L}+\mathrm{M} \beta \mathrm{L} .12$ disc test is a good screening test for rapid identification of type of $\beta$ lactamases.
\end{abstract}

\section{Introduction}

An alarming rise in the rates of the antibiotic resistance has now become a serious and an increasingly common public health concern, with severe implications, especially in the intensive care units. The $\beta$-lactam antibiotics are among the most frequently prescribed antibiotics world-wide because of their efficacy, broad spectra and low toxicity.

A variety of mutated forms of $\beta$-lactamases like the extended spectrum beta lactamases
(ES $\beta \mathrm{Ls})$, AmpC $\beta$-lactamases (AmpC $\beta \mathrm{L})$ and metallo- $\beta$-lactamases (M $\beta \mathrm{L})$ have evolved due to the selective pressure generated by the indiscriminate use of these antibiotics. This has emerged as the most worrisome resistance mechanism posing a therapeutic challenge to the health care settings as they are capable of hydrolyzing a wide range of $\beta$-lactam antibiotics, notably the extended-spectrum penicillins, third and fourth generation cephalosporins, and the carbapenems (Oberoi L. et al., 2013) 
The confirmed ES $\beta$ L-producing isolates should be reported as resistant to all penicillins, cephalosporins, and aztreonam to avoid therapy with antibiotics that may be clinically ineffective but bacteria producing these enzymes may not be recognized as they are falsely susceptible in routine tests as a result patients start receiving ineffective antibiotics which contributes to the spread of the pathogens with hidden resistance. Tests based on ceftazidime and cefotaxime tested alone and in combination with clavulanate are useful in ESBL detection but they may yield false positive results with Klebsiella pneumonia carbapenemase (KPCs) and hyper produced $\mathrm{K} 1 \beta$-lactamaes $(\mathrm{K} 1 \beta \mathrm{L})$ and false negative results with isolates that coproduce a high level of AmpC $\beta \mathrm{L}$. Thus, detection of AmpC $\beta L$ especially in these has its own importance because they have been associated with false ES $\beta$ L negative results. Carbapenems are generally used to treat $\mathrm{ES} \beta \mathrm{L} / \mathrm{AmpC} \beta \mathrm{L}$ - associated infections, so it is also important that reduced carbapenem susceptibility of ESBL-positive isolates is not ignored simultaneously as it may indicate carbapenemase production, which contraindicates carbapenem therapy too.

Their detection is a crucial infection control issue because they are often associated with extensive, sometimes total, antibiotic resistance and more-resistant organisms can be vectors responsible for carbapenemase transmission to members of the families in which the resistance mechanism is not recognized (Kenneth et al., 2010)

We conducted this study to detect different resistance phenotypes of beta lactamases among isolates of family Enterobacteriaceae, Pseudomonas spp. and Acinetobacter spp. in our set up and to evaluate a novel 12 disc test (a single plate method) as a screening test to detect the presence of any kind of beta lactamase or its co producers.

\section{Material and Methods}

The study was conducted in the department of Microbiology, SMS medical College, Jaipur from the period of March 2014 to April 2014. A total of 339 consecutive, non repetitive clinical isolates of gram negative bacilli (GNB), belonging to family Enterobacteriaceae, Pseudomonas spp. and Acinetobacter spp from different clinical samples, which were received from various wards and ICUs were identified by the standard microbiological tests (Collee, et al., 1996). The routine antimicrobial susceptibility pattern of the isolates was determined by the Kirby Bauer disc diffusion method according to the CLSI guidelines (CLSI 11 ${ }^{\text {th }}$ edn, 2012). A 12 disc test (Schreckenberger et al) was put on 300 isolates that showed susceptibility pattern as Intermediate/Resistant to ceftazidime/ ceftriaxone or aztreonam. Though, Schreckenberger PC. et al., recommend the 12 disc test for family Enterobacteriaceae only, but we have used it on Pseudomonas and Acinetobacter spp. too as they are also commonly isolated in our set up. The reference strains, ES $\beta \mathrm{L}$ positive Klebsiella pneumonia ATCC 700603 and E. Coli ATCC 25922 were included in the study as controls.

\section{Disc Test}

(Schrekenberger et al): Mueller Hinton agar (MHA) in $150 \mathrm{~mm}$ diameter plate was used. Following antibiotic discs (Hi media labs Pvt Ltd) were placed as shown in fig 1:

Aztreonam (30), Ceftazidime (30), Ceftazidime + clavulante (30/10), Cefotaxime (30), Cefotaxime +clavulante (30/10), Cefoxitin (30), Cefotetan (30), Ceftriaxone (30), Cefepime (30), Ertapenem (10), Imipenem(10), Meropenem (10).

Interpretation criteria used for the screening test (Schrekenberger et al): 
1. An isolate showing an increase in zone size of $\geq 5 \mathrm{~mm}$ with clavulanate compared with plain ceftazidime/cefotaxime disks is interpreted as ES $\beta \mathrm{L}$ positive.

2. AmpC $\beta \mathrm{L}$ is detected when the isolate shows resistance to first, second and third generation cephalosporins, the beta lactam inhibitor drug and cephamycins, but susceptibility to Cefepime disk. In addition to the above, high level AmpC $\beta \mathrm{L}$ producers are resistant to Monobactem (Aztreonam).

3. Carbapenemase production is indicated by resistance to the Carbapenems (Meropenem/ Imipenem / Etrapenem).

4. K1 $\beta L$ : Sensitive to cefoxitin, No Clavulanic Effect, Resistant to Aztreonam and sensitive to ceftazidime.

After performing the 12 disc test, all the isolates were subjected to following CLSI recommended phenotypic tests to compare the results with the screening test. For ESBL detection, double disc synergy test (Giriyapur RS. et al., 2011), for MBL/KPC detection, modified Hodge test (Amjad et al., 2011) and E-test method using EzyMIC TM Strips (Hi Media Laboratories Pvt. Ltd.) were used (Mobashshera et al., 2015). Along with these, AmpC disc test (Singhal S. et al., 2005) for AmpC $\beta L$ detection was used and all of them were considered as confirmatory tests in our study.

Result: Out of the 339 isolates of Enterobacteriaceae, Pseudomonas spp and Acinetobacter spp, 300 (88.4\%) isolates were found to be producing any of the beta lactamases. Among these 300 isolates, $144(45 \%)$ were E.Coli, $56 \quad(18.6 \%)$
Enterobacter spp, 32 (10.6\%) Pseudomonas spp, 20(6.6\%) were Klebsiella spp, 20 (6.6\%) Acinetobacter spp, 12(4\%) Proteus spp, 12(4\%) Citrobacter spp and 4(1.3\%) Hafnia spp.

Table1 shows organism wise distribution of various beta lactamases.

All isolates of Hafnia spp (04) were found to be ES $\beta \mathrm{L}$ producers (100\%), whereas maximum number of $\mathrm{AmpC} \beta \mathrm{L}$ and carbapenemase producers (CPs) were from Acinetobacter spp (80\% each) in this study.

Table 2 depicts the results of 12 disc test.

Distribution of different types of beta lactamases detected by the confirmatory tests used in the study is shown in table 3. Out of 300 screened isolates, total ES $\beta \mathrm{L}$ producers were found to be 104 (34.6\%), K1 $\beta \mathrm{L} 8$ (2.6\%), AmpC $\beta \mathrm{L}$ producers 156 $(52 \%)$ and CPs were 132 (44\%). Out of the 132 CPs, $80(60.6 \%)$ strains were sensitive to Imipenem and showed resistance to Meropenem, whereas $8(6 \%)$ strains which were sensitive to Meropenem exhibited resistance to Imipenem. $84(24 \%)$ isolates were found to be co- producers of these beta lactamases.

When the results of 12 disc test were compared with the confirmatory tests from tables 2 and 3, ES $\beta \mathrm{L}, \mathrm{AmpC} \beta \mathrm{L}$ and high level AmpC $\beta \mathrm{L}$ producers were found to be exactly same by both the tests i.e 64 (21.6\%), 8 (2.6\%) and 76 (25.3\%) respectively. Out of $108 \quad(36 \%)$ CPs detected by 12 disc test, $60(20 \%)$ were confirmed as CPs and $44(14.6 \%)$ turned out to be co producers of AmpC $\beta L+C P$ by confirmatory tests. Chromosomal AmpC $\beta \mathrm{L}$ $+\mathrm{ES} \beta \mathrm{L}$ co producers detected by 12 disc test were $8(2.6 \%)$, whereas by confirmatory test $12(4 \%)$. 
12 disc test detected $28(9.3 \%)$ as ES $\beta \mathrm{L}+\mathrm{CP}$ co producers, out of these, only $12(4 \%)$ turned out to be $\mathrm{ES} \beta \mathrm{L}+\mathrm{CP}$ and rest 16 (5.3\%) were $\mathrm{ES} \beta \mathrm{L}+\mathrm{AmpC} \beta \mathrm{L}+\mathrm{CP}$ by confirmatory methods..

$8(2.6 \%)$ isolates which were reported as $\mathrm{CP}$ by 12 disc test were found to be KPC by confirmatory tests. K1 $\beta \mathrm{L}$ could only be detected by 12 disc test and were 8 (2.6\%). They were found to be negative for any of the beta lactamases by confirmatory tests.

\section{Results and Discussion}

Clinical laboratories have been facing problems in detection of the enzymes ES $\beta$ Ls, AmpC $\beta L$ and M $\beta$ Ls since they were discovered. Also, the co expression of these enzymes makes their detection more complicated. Confusion still persists in the choice of optimal test methods, their reporting conventions and the importance of resistance mechanism. Failure of detection of these enzymes lead to therapeutic failures and uncontrolled spread further.

We have undertaken this study to detect different beta lactamases among isolates of Enterobacteriaceae, in our set up. Also, we have evaluated a novel 12 disc test (a single plate method) as a screening test to detect the presence of any kind of beta lactamase or its co producers and compared its results with standard phenotypic methods used as confirmatory tests.

In the present study, the prevalence of ES $\beta \mathrm{L}$ positive strains was found to be 104/339 $(30.6 \%)$. Studies done previously had reported an overall prevalence of ESBL varying from $33.86 \%$ to $64.8 \%$.(Valsan et al., 2013, Wadekar et al., 2013, Sinha et al., 2008, Vijaya et al., 2014) It is a well known fact that ES $\beta L$ positive strains often show false susceptibility results on standard disc diffusion method. However, their spread is so extensive that all laboratories should include their detection by means of special tests, along with the routine testing by CLSI recommended methods.

The prevalence of AmpC $\beta \mathrm{L}$ positive strains in our study period was found to be $156 / 339$ (46.0\%). Others have reported $50.9 \%$ (Chatterjee et al., 2010), 24\% (Sinha et al., 2008) $14.0 \%$ (Vijaya et al., 2014) and $11.1 \%$ (Laghawe et al., 2012). This shows a wide variation in the prevalence of beta lactamases from region to region or even from hospital to hospital. Prevalence of $A m p C \beta L$ as found in our present study is almost double of what we had reported seven years back (24\%) in a study done under same set up (Sinha P. et al., 2008). Efforts to detect AmpC $\beta L$ enzymes in GNBs are largely non-existent. Undoubtedly this is due in part to the lack of standard guidelines for detecting AmpC $\beta \mathrm{L}$ producing isolates (Laghawe et al., 2012)

Overall 132/339 (38.9\%) isolates were found to be M $\beta \mathrm{L}$ producers in our study. This is higher in comparison to some studies (Valsan et al., 2013, Wadekar MD. et al., 2013, Vijaya et al., 2014), but correlates well with the study done by Chatterji SS. et al., (2010) who had reported a positivity rate of $41.7 \%$. Production of MBL has tremendous therapeutic consequences since these organisms also carry multidrug resistance genes and the only viable option remains the potentially toxic Polymyxin B and Colistin (Wadekar et al., 2013). In the present study, we found large number of $\mathrm{M} \beta \mathrm{L} / \mathrm{KPCs}$ to be sensitive to Imipenem but resistant to meropenem i.e. $80(60.6 \%)$, whereas $8(6 \%)$ showed vice versa results. Renu et al., (2010) had reported 20\% Imipenem sensitive M $\beta$ Ls in her study. These carbapenem susceptible organisms with hidden M $\beta \mathrm{L}$ genes can spread unnoticed in hospitals if such isolates are 
reported as sensitive without screening for the presence of M $\beta$ Ls. Screening of only Imipenem resistant organisms for $\mathrm{M} \beta \mathrm{L}$ is insufficient and screening of all the Imipenem susceptible isolates creates unnecessary work with a lower yield. Hence, some criterion is needed to select out Imipenem susceptible isolates for $\mathrm{M} \beta \mathrm{L}$ screening. In 12 disc test, three carbapenems i.e. Imipenem, Meropenem and Ertapenem are used so as not to miss any of the M $\beta L$ s.

Co existence of ES $\beta \mathrm{L}$ and AmpC $\beta \mathrm{L}$ was seen in $12(4 \%)$ of the 300 isolates. Others have reported 8\% (Sinha P. et al., 2008) and $16.7 \%$ (Vijaya et al., 2014). ES $\beta \mathrm{L}$ with $\mathrm{M} \beta \mathrm{L} / \mathrm{KPC}$ were seen in $12(4 \%)$ isolates and this was similar to the study done by Laghave et al., 2012, who has reported $4.86 \%$ of co existence. Vijaya et al., (2014) and Salimi et al., (2013) have also reported coexistence of $1.5 \%$ and $12.5 \%$ respectively. AmpC $\beta \mathrm{L}+\mathrm{M} \beta \mathrm{L}$ were seen among 44 $(14.6 \%)$ isolates in our study, whereas Salimi F. et al., (2013) and Vijaya et al., 2014 have reported in $81 \%$ and $1.5 \%$ isolates respectively. All three i.e. ES $\beta \mathrm{L}+$ $\mathrm{M} \beta \mathrm{L}+\mathrm{AmpC} \beta \mathrm{L}$ were seen in $16(5.3 \%)$ isolates in our study. Others have reported $23.7 \%$ (Chatterjee et al., 2010) and 1.5\% (Salimi et al., 2013). Thus, there is high level of coexpression of these beta lactamases as resistance mechanism, which cannot be ignored and their timely detection needs to be stressed upon.

In 12 disc test, two sets of double discs (CAZ - CAZ + Clav and CTX - CTX + Clav) for $\mathrm{ES} \beta \mathrm{L}$ and $\mathrm{K} 1 \beta \mathrm{L}$ screening, two discs (Cefotetan and Cefoxitin) for AmpC $\beta \mathrm{L}$ screening and three drug discs (Imipenem, Meropenem and Ertapenem) for screening of $\mathrm{M} \beta \mathrm{L} / \mathrm{KPC}$ were used so that none of the beta lactamase is missed in screening. When the results of 12 disc test were compared with the confirmatory tests as shown in tables 3 and 4, no discrepancy was found in detection of ES $\beta \mathrm{L}, \mathrm{AmpC} \beta \mathrm{L}$ and high level AmpC $\beta$ L.

There are currently no CLSI approved methods to detect chromosomal or plasmid mediated AmpC gene resistance but as per the recommendations of 12 disc test, 84/92 (91.3\%) plasmid mediated AmpC $\beta \mathrm{L}$ and 8/92 (8.6\%) chromosomal AmpC $\beta L$ were detected in our study. Chromosomal AmpC beta-lactamases can be produced inducibly or constitutively and inducible expression of the AmpC gene occurs when the enzyme is produced at a high level for example under exposure to inducing agents, such as Cephamycins (ie. Cefoxitin), Ampicillin and Carbapenems (i.e. Imipenem, Meropenem, Ertapenem). Induction is temporary and may be reversed when the antibiotic inducer is removed. In some organisms, mutations occur that cause the AmpC $\beta \mathrm{L}$ gene to become permanently expressed at high levels. These organisms are termed permanently de-repressed mutants (Schreckenberger et al.,)

Out of 108 (36\%) pure CPs, detected by 12 disc test, only 60 (20\%) came out to be CPs by confirmatory methods and 44 (14.6\%) turned out to be AmpC $\beta L+$ CP. Similarly 12 disc test detected $28(9.3 \%)$ isolates as ES $\beta L+C P$, out of which only $12(4 \%)$ turned out to be $\mathrm{ES} \beta \mathrm{L}+\mathrm{CP}$ and rest 16 $(5.3 \%)$ were ES $\beta \mathrm{L}+\mathrm{AmpC} \beta \mathrm{L}+\mathrm{CP}$ by confirmatory tests. Such discrepancy may not change the therapeutic outcome as the treatment given for AmpC $\beta \mathrm{L}+\mathrm{CP}$ and for $\mathrm{ES} \beta \mathrm{L}+\mathrm{AmpC} \beta \mathrm{L}+\mathrm{CP}$ is same as given for $\mathrm{CP}$ alone. 
Table.1 Organism wise prevalence of different kind of beta lactamases.

\begin{tabular}{lllll}
\hline & ES $\beta \mathrm{L}$ & AmpC $\beta \mathrm{L}$ & M $\beta \mathrm{L} / \mathrm{KPC}$ & K1 $\beta \mathrm{L}$ \\
\hline E. coli (144) & $56(38.8 \%)$ & $64(44.4 \%)$ & $52(36.1 \%)$ & 0 \\
Citrobacter (12) & 0 & $8(66.6 \%)$ & $4(33.3 \%)$ & 0 \\
Enterobacter (56) & $12(21.4 \%)$ & $40(71.4 \%)$ & $28(50 \%)$ & $4(7.1 \%)$ \\
Pseudomonas (32) & $16(50 \%$ & $20(62.5 \%)$ & $20(62.5 \%)$ & 0 \\
Acinetobacter (20) & $4(20 \%)$ & $16(80 \%)$ & $16(80 \%)$ & 0 \\
Klebsiella (20) & $4(20 \%)$ & $8(40 \%)$ & $12(60 \%)$ & 0 \\
Proteus (12) & $8(66.6 \%)$ & 0 & 0 & $4(33.3 \%)$ \\
Hafnia (4) & $4(100 \%)$ & 0 & 0 & 0 \\
\hline Total (300) & $104(34.6 \%$ & $156(52 \%)$ & $132(44 \%)$ & $8(2.6 \%)$ \\
\hline ES $\beta \mathrm{L}$, AmpC $\beta \mathrm{L}, \mathrm{M} \beta \mathrm{L} / \mathrm{KPC}$ as detected by confirmatory tests and K1 $\beta \mathrm{L}$ by 12 disc test. &
\end{tabular}

Table. 2 Results of 12 Disc Test ( $\mathrm{n}=300)$.

\begin{tabular}{ll}
\hline Type of beta lactamase & Number (percentage) \\
\hline ES $\beta \mathrm{L}$ & $64(21.3 \%)$ \\
K1 $\beta \mathrm{L}$ & $8(2.6 \%)$ \\
Plasmid mediated AmpC & \\
High level AmpC $\beta \mathrm{L}$ & $76(25.3 \%)$ \\
AmpC $\beta \mathrm{L}$ & $08(2.6 \%)$ \\
Chromosomal AmpC $\beta \mathrm{L}+\mathrm{ES} \beta \mathrm{L}$ & \\
With high level AmpC $\beta \mathrm{L}$ & $08(2.6 \%)$ \\
With AmpC $\beta \mathrm{L}$ & 0 \\
Carbapenemase & $108(36 \%)$ \\
ES $\beta \mathrm{L}+\mathrm{CP}$ & $28(9.3 \%)$ \\
\hline
\end{tabular}

Table.3 Results of confirmatory tests $(n=300)$.

\begin{tabular}{ll}
\hline Type of beta lactamase & Number (percentage) \\
\hline ES $\beta \mathrm{L}$ & $64(21.3 \%)$ \\
High level AmpC $\beta \mathrm{L}$ & $76(25.3 \%)$ \\
AmpC $\beta \mathrm{L}$ & $08(2.6 \%)$ \\
M $\beta \mathrm{L}$ & $52(17.3 \%)$ \\
KPC & $8(2.6 \%)$ \\
ES $\beta \mathrm{L}+\mathrm{AmpC} \beta \mathrm{L}$ & $12(4 \%)$ \\
ES $\beta \mathrm{L}+\mathrm{M} \beta \mathrm{L}$ & $12(4 \%)$ \\
AmpC $\beta \mathrm{L}+\mathrm{M} \beta \mathrm{L}$ & $44(14.6 \%)$ \\
ES $\beta \mathrm{L}+\mathrm{AmpC} \beta \mathrm{L}+\mathrm{M} \beta \mathrm{L}$ & $16(5.3 \%)$ \\
\hline
\end{tabular}


Fig.1 Template for 12 disc test

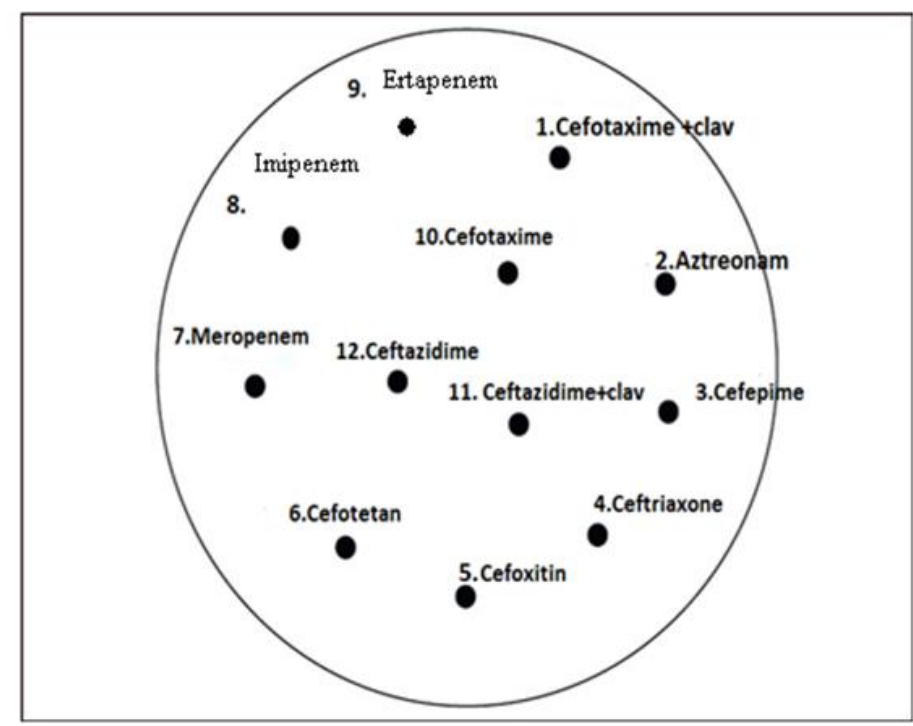

Fig.2 12 disc test: Clavulanate enhancement present - ES $\beta L$ positive. Resistance to ertapenem and meropenem indicate carbapenemase production and Cefoxitin, cefotetan and cefepime are resistant. Confirmatory test: $\mathrm{ES} \beta \mathrm{L}+\mathrm{AmpC} \beta \mathrm{L}+\mathrm{M} \beta \mathrm{L}$.

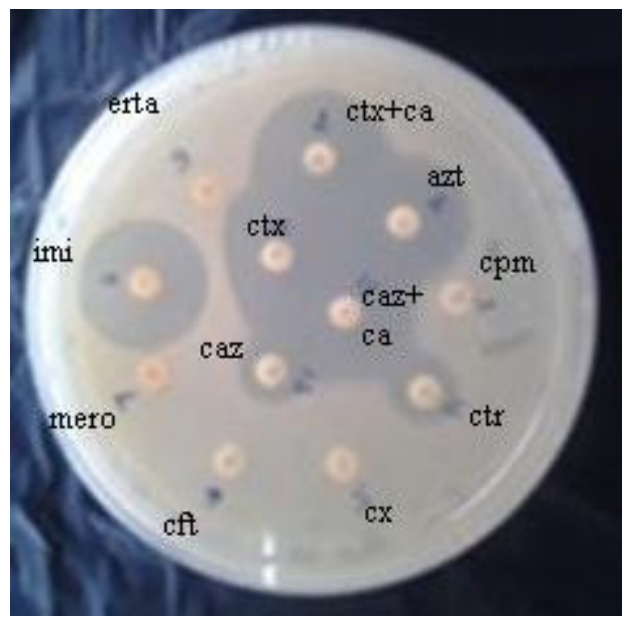

Ctx: cefotaxime, caz: ceftazidime, caz+ clavulanic acid, Ctx + ca: cefotaxime + clavulanic acid, azt: aztreonam, cpm: cefepime, ctr: ceftriaxone, Cx: cefoxitin, cft: cefotetan, mero: meropenem, Erta: ertapenem, 
Fig.3 12 disc test: Keyhole towards clavulanic acid indicates ES $\beta$ L. Cefepime sensitive and Cefoxitin/Cefotetan resistant indicate AmpC $\beta \mathrm{L}$; Imipenem and Meropenem both are sensitive but Etrapenem is resistant, indicates probability of carbapenemase production.

Confirmatory tests: $\mathrm{ES} \beta \mathrm{L}+\mathrm{AmpC} \beta \mathrm{L}$

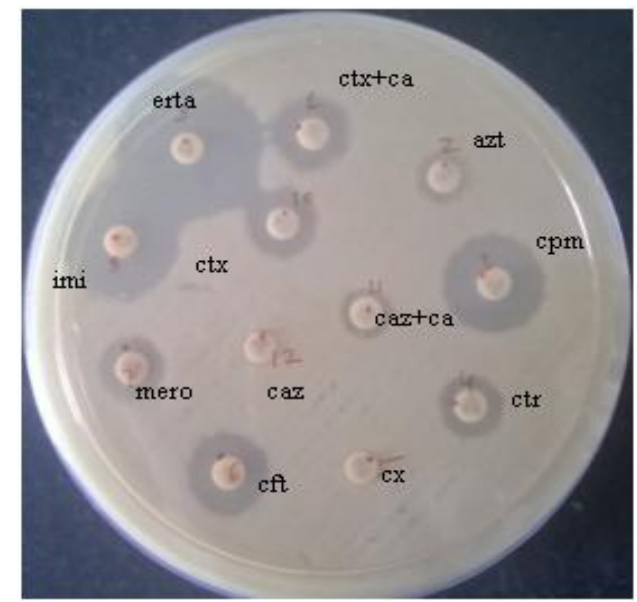

Ctx: cefotaxime, caz: ceftazidime, caz+ clavulanic acid, Ctx+ca: cefotaxime + clavulanic acid, azt: aztreonam, cpm: cefepime, ctr: ceftriaxone, Cx: cefoxitin, cft: cefotetan, mero: meropenem, Erta: ertapenem,

12 disc test detected $8(2.6 \%)$ strains as Chromosomal AmpC $\beta \mathrm{L}+\mathrm{ES} \beta \mathrm{L}$ со producers whereas it was $12(4 \%)$ by confirmatory test. This little discrepancy may be the result of masking effect of AmpC $\beta \mathrm{L}$ on clavulanate enhancement which is used for detection of ES $\beta \mathrm{L}$.

KPCs which could be detected only by the confirmatory tests were $8(2.6 \%)$ and were reported as $\mathrm{CP}$ by 12 disc test. With 12 disc test, we are unable to differentiate KPC from M $\beta$ L. We could detect K1 $\beta \mathrm{L} 8(2.6 \%)$ which were considered as negative for any of the beta lactamases by confirmatory tests. The $\mathrm{K} 1$ enzyme is predominantly a penicillinase that can also significantly hydrolyze Aztreonam, Cefuroxime and Ceftriaxone and has weak activitiy against Cefotaxime or Ceftazidime. A distinctive feature of hyperproducers of $\mathrm{K} 1$ is its greater activity against Ceftriaxone over Cefotaxime and against Aztreonam over Ceftazidime (Schreckenberger P. et al) Unfortunately, there are currently no CLSI approved methods to detect $\mathrm{K} 1$ beta lactamases too.

In conclusion, microbiology laboratories must be able to detect resistant pathogens in a timely manner, especially those that are falsely susceptible in vitro to drugs that may be considered for therapy of infected patients so as to modify the treatment of the patient and prevent the dissemination of resistant strains further. We found that the 12 disc test is a very useful, sensitive and cost effective test which can be used in routine clinical microbiological laboratories for screening family enterobacteriaceae and the routinely isolated non fermenters, especially in the set ups where the automated systems or molecular facilities are not available. This will reduce the chances of missing any type of beta lactamases thereby reporting them as susceptible instead of resistant. The drawback of our study is that we have not 
confirmed our results with molecular methods but except for AmpC $\beta \mathrm{L}$ and $\mathrm{K} 1 \beta \mathrm{L}$ detection, we have used only CLSI recommended methods as confirmatory tests.

\section{References}

Oberoi, L., Singh, N., Sharma, P., Aggarwal, A. 2013. ESBL, MBL and Ampc $\beta$ Lactamases Producing Superbugs - Havoc in the Intensive Care Units of Punjab India. J. Clin. Diagn. Res., 7(1): 70-3.

Kenneth, S., Thomson. 2010. ExtendedSpectrum- $\beta$-Lactamase, AmpC, and Carbapenemase Issues. J. Clin. Microbiol., 48(4): 1019-25.

Collee, J.G., Fraser, A.G., Marmion, B.P., Simmons, A., Mackie, McCartney. 1996. Practical medical microbiology, 14th ed. London: Churchill Livingstone, 361(81): 417 23.

CLSI. 2012. Performance standards for antimicrobial disc susceptibility tests; approved standard - 11th ed., CLSI, document M02-A11.

Schreckenberger, P.C., Rekasius, V. Phenotypic Detection of $\beta$ Lactamase Resistance in GramNegative Bacilli: Testing and Interpretation Guide (Rev. 2-21-12).

Giriyapur, R.S., Nandiha, N.W., Krishna, B.V.S., Patil, A.B., Chandrasekhar, M.R. 2011. Comparison of Disc Diffusion Methods for the Detection of Extended-Spectrum Beta Lactamase- producing Enterobacteriaceae. J. Lab. Physicians, 3(1): 33-6.

Amjad, A., Mirza, I.A., Abbasi, S.A., Farwa, U., Malik, N., Zia, F. 2011. Modified Hodge test: A simple and effective test for detection of carbapenemase production. Iran J. Microbiol., 3(4):
189-93.

Mobashshera, T., Aruna, K. 2015. Phenotypic and Molecular Characterization of MBL Genes among Uropathogens Isolated in Mumbai City. BMRJ, 5(4): 368-83.

Singhal, S., Mathur, T., Khan, S., Upadhyay, D.J., Chugh, S., Gaind, R., et al. 2005. Evaluation of methods for AmpC Beta -lactamase in gram negative clinical isolates from tertiary care hospitals. Indian J. Med. Microbiol., 23: 120-4.

Valsan, C., Chinnan, J.P., Sathiavathy, K.A. 2013. Phenotypic detection of $\beta$ lactamases in enterobacteriaceae using a 12-disk procedure. J. Acad. Clin. Microbiol., 15: 7-10.

Wadekar, M.D., Anuradha, K., Venkatesha, D. 2013. Phenotypic detection of ESBL and MBL in clinical isolates of Enterobacteriaceae. Int. J. Curr. Res. Aca. Rev., 1(3): 89-95.

Sinha, P., Sharma, R., Rishi, S., Sood, S., Pathak, D. 2008. Prevalence of extended spectrum beta lactamase and AmpC beta lactamase preoducers among Escherichia Coli isolates in tertiary care hospital in Jaipur. Indian J. Pathol. Microbiol., 51: 367-9.

Vijaya, D., Dhanalakshmi, A. 2014. Prevalence of ESBL, AmpC and Carbapenemase among Gram Negative Bacilli isolated from Clinical Specimens. Am. J. Life Sci., 2(2): 76-81.

Chatterjee, S.S., Karmacharya, R., Madhup, S.K., Gautam, V., Das, A., Ray, P. 2010. High prevalence of coexpression of newer $\beta$-lactamases (ESBLs, Amp-C- $\beta$-lactamases, and metallo- $\beta$-lactamases) in gramnegative bacilli. Ind. J. Med. Microbiol., 28(3): 267-8. 
Laghawe, A.R., Jaitly, M.S., Neelam, K., Vilas, T. 2012. Prevalence of AmpC Beta- lactamase in Gram- negative bacilli. J. Pharm. Biomed. Sci., 20(07): 1-4.

Renu, G., Rajeev, T., Smita, S. 2010. The Existence of Metallo Beta Lactamases in Carbapenem Susceptible Gram Negative Bacilli: A Cause for Concern. J. Clin. Diagn. Res., (4): 2679-84.
Salimi, F., Eftekhar, F. 2013. Coexistence of AmpC and Extended-Spectrum $\beta$ lactamases in Metallo $\beta$-Lactamase producing Pseudomonas aeruginosa Burn Isolates in Tehran. Jundishapur J. Microbiol., 6(8): 7178.

Schreckenberger, P., Rekasius, V. Detecting Resistance to Beta Lactams in GramNegative Bacilli. Available at: http://www.hardydiagnostics.com/art icles/antibiotic-resistance.pdf.

\section{How to cite this article:}

Parul Sinha, Pallawi Goyal, Rajni Sharma, Aruna Vyas and Maheshwari, R.K. 2016. Evaluation of a 12 Disc Test for Phenotypic Detection of $\beta$ - lactamases Resistance in Gram Negative Bacilli. Int.J.Curr.Microbiol.App.Sci. 5(6): 105-114. doi: http://dx.doi.org/10.20546/ijcmas.2016.506.013 